\title{
Diagnostic performance of machine learning applied to texture analysis-derived features for breast lesion characterisation at automated breast ultrasound: a pilot study
}

\author{
Magda Marcon ${ }^{*+} \mathbb{D}$, Alexander Ciritsis ${ }^{\dagger}$, Cristina Rossi, Anton S. Becker, Nicole Berger, Moritz C. Wurnig,
} Matthias W. Wagner, Thomas Frauenfelder and Andreas Boss

\begin{abstract}
Background: Our aims were to determine if features derived from texture analysis (TA) can distinguish normal, benign, and malignant tissue on automated breast ultrasound (ABUS); to evaluate whether machine learning (ML) applied to TA can categorise ABUS findings; and to compare ML to the analysis of single texture features for lesion classification.

Methods: This ethically approved retrospective pilot study included 54 women with benign $(n=38)$ and malignant $(n=32)$ solid breast lesions who underwent ABUS. After manual region of interest placement along the lesions' margin as well as the surrounding fat and glandular breast tissue, 47 texture features (TFs) were calculated for each category. Statistical analysis (ANOVA) and a support vector machine (SVM) algorithm were applied to the texture feature to evaluate the accuracy in distinguishing (i) lesions versus normal tissue and (ii) benign versus malignant lesions.
\end{abstract}

Results: Skewness and kurtosis were the only TF significantly different among all the four categories $(p<0.000001)$. In subsets (i) and (ii), a maximum area under the curve of 0.86 (95\% confidence interval $[\mathrm{Cl}]$ 0.82-0.88) for energy and 0.86 (95\% Cl 0.82-0.89) for entropy were obtained. Using the SVM algorithm, a maximum area under the curve of 0.98 for both subsets was obtained with a maximum accuracy of $94.4 \%$ in subset (i) and $90.7 \%$ in subset (ii).

Conclusions: TA in combination with ML might represent a useful diagnostic tool in the evaluation of breast imaging findings in ABUS. Applying ML techniques to TFs might be superior compared to the analysis of single TF.

Keywords: Breast neoplasms, Machine learning, Ultrasonography

\section{Key points}

- Analysis of texture features on automated breast ultrasound can help to categorise imaging findings.

- Machine learning can be applied to texture features to categorise breast lesions.

- Machine learning performs better than the analysis of single texture features.

\footnotetext{
* Correspondence: magda.marcon@usz.ch

${ }^{\dagger}$ Magda Marcon and Alexander Ciritsis contributed equally to this work. Institute of Diagnostic and Interventional Radiology, University Hospital Zurich, Raemistrasse 100, 8091 Zurich, Switzerland
}

\section{Background}

In women with dense breast tissue, the combined use of mammography and hand-held ultrasound (HHUS) in breast cancer screening boosts breast cancer detection rate with additionally detected 2-4 cancers per 1,000 women [1-4]. However, the use of HHUS in the screening setting remains controversial due to its inherent limitations including the lack of standardisation and the necessary level of operator experience $[4,5]$. In recent years, automated breast ultrasound (ABUS) has been introduced to overcome some of HHUS limitations. ABUS provides technique standardisation via the acquisition of standardised views as well as scanning parameters and 
resolves the issue of operator subjectivity and variation [6]. Nevertheless, interpretation of imaging findings remains highly dependent on reader skills and experience. Standardised acquisition in terms of scanning parameters (e.g., focus, gain) offers the opportunity to apply tools for image analysis that can support the characterisation of imaging findings.

Texture analysis (TA) is an integral part of the emerging field of radiomics and allows a quantitative and objective assessment of tissue heterogeneity by evaluating the distribution and relationship of pixel or voxel grey levels in the image $[7,8]$. In most of the cases, methods based on statistical analysis are used to represent the interdependence of grey-level values. TA applied to computed tomography and magnetic resonance imaging has already shown promising results in predicting pathologic features, prognosis and response to therapy for various diseases and body compartments and can potentially be used in ABUS imaging for lesion analyses [9-16]. Moreover, machine learning (ML) can be applied to data from TA such that algorithms are trained to learn specific patterns and categorise the imaging findings [17].

In this context, the primary purpose of our study was to determine if features derived from TA can be used to distinguish normal tissue, malignant and benign solid lesions in ABUS. Second, we evaluated whether ML applied to TA data can accurately categorise ABUS findings. Third, we compared ML to the analysis of single texture features to categorise ABUS finding based on TA.

\section{Methods}

\section{Study subjects}

The local ethics board approved this retrospective study ("Kantonale Ethikkommission Zurich"; Approval Number: 2016-00064). The need for informed consent was waived. Between December 2015 and June 2017, all women with at least one histologically proven malignant lesion $(n=27$; median age 54 years; range $30-85$ years) who underwent ABUS imaging were identified from the hospital database (University Hospital Zurich). An equal number of women $(n=27)$ with at least one benign solid lesion (median age 44 years; age range 27-73 years) who underwent ABUS during the same study period were also included. In case of a malignant lesion, the histological type was collected. All benign solid lesions had to be either histopathologically proven fibroadenomas or stable lesions with a followup of at least 24 months. ABUS was performed in addition to mammography in 39 women with American College of Radiology breast density category $c$ or $d$ [18] undergoing screening examination and as unique imaging examination in 15 women younger than 40 years undergoing routine controls. None of the patients was symptomatic or had strong family history of breast cancer (i.e., no BRCA1 or BRCA2 mutation carriers, no first-degree relatives of BRCA1 or BRCA2 mutation carriers, and no women with three or more events of ovarian cancer or male breast cancer or breast cancer in women younger than 60 years in first- or second-degree relatives in either maternal or paternal line). The maximum diameter in ABUS was annotated for all lesions.

\section{ABUS examination}

Images were acquired with ABUS (Invenia ${ }^{\mathrm{mm}}$ Automated Breast Ultrasound System, General Electric Healthcare, Sunnyvale, CA, USA) using a C 15-6XW reverse curve, 5$14 \mathrm{MHz}$ transducer with an aperture length of $15.3 \mathrm{~cm}$, a transducer travel distance of $16.9 \mathrm{~cm}$, and a depth up to 5 $\mathrm{cm}$. An abundant layer of water-based lotion is applied to the breast in order to maximise the coupling between the transducer and the skin. The standard acquisition included three volumes per breasts, so-called anteroposterior, lateral, and medial in order to guarantee coverage of the entire breast. Slices had a thickness of $0.5 \mathrm{~mm}$. Volume acquisitions were performed in the axial plane, and the 3D reconstructions in the sagittal and coronal planes were automatically provided using a dedicated workstation.

\section{Image selection and texture analysis}

All axial images encompassing the lesion in the three volumes were analysed separately. Images in which the visibility of the lesion was altered because of artefacts (i.e., inadequate compression during the volume acquisition or inadequate lotion with impaired acoustic coupling at the contact surface between the transducer and the skin) were excluded from the analysis $(n=63)$. These images were in general only part of a patient examination (e.g., two to three images in one of the volumes) and did not determine any complete exclusion of patients. Normal fat and fibroglandular tissue were evaluated in two additional, arbitrarily selected images for each patient, usually from the upper outer quadrant (in patients with malignant lesions in the contralateral breast) in order to evaluate the texture features of normal breast tissue. The image selection was performed by a radiologist with 8 years of experience in breast imaging and 3 years of experience in ABUS imaging.

TA was performed in MATLAB (v2016b, The MathWorks Inc., Natick, MA, USA) with an established routine-based procedure, as already described $[19,20]$. A region of interest (ROI) was drawn freehand by a radiologist (with 8 years of experience in breast imaging) who delineated the outer edge of the lesion or the maximal continuous area of fibroglandular or fat tissue included in a single image. A second radiologist (with 7 years of experience in breast imaging) performed the same evaluation in five benign and five malignant lesions. In order to minimise intrascanner 
effects, ROI content normalisation between the mean and three standard deviations $(\mu \pm 3 \sigma)$ was performed as a first step of the TA [21,22]. Subsequently, 47 features were computed [9] (Table 1). The first order features (entropy, variance, skewness and kurtosis) were directly extracted from the histogram of all grey levels in the ROI. The second and high-order features were derived from the respective grey-level matrices (i.e., grey-level co-occurrence matrix [GLCM]; grey-level run length matrix [GLRLM] and grey-level size zone matrix [GLSZM]) and included more information concerning grey-level distribution by accounting for the relative position of each pixel with respect to the other pixels of the image $[9,23]$.

\section{Machine learning}

\section{Data preparation}

Preprocessing and preparation of the dataset for ML were performed with routines written in Python and Scikit-learn (www.scikit-learn.org, release 0.18.1). All features obtained from texture analyses were standardised for the whole dataset using the Scikit-learn-embedded "StandardScaler" class, by removing the mean and scaling the data to unit variance. To account for multiclass classification, the dataset with four classes (malignant lesions, benign solid lesions, fat tissue, glandular tissue) was split into two balanced subdatasets, each consisting of two classes: (i) solid lesions versus normal fat and glandular tissue and (ii) malignant lesions versus benign solid lesions. To measure the unbiased performance of the classifier each sub-dataset was randomly shuffled and split in a stratified manner into training and validation partition, with a ratio of $0.8-0.2$. The validation partition was excluded from the training process, serving as "unseen" real-world data. Thereby, special attention was put on the fact that each TA dataset in each validation partition was acquired from an individual patient.

\section{Support vector machine classifier}

An ML model based on the support vector machine (SVM) algorithm with radial basis decision function and fivefold cross-validation was implemented using Scikit-learn. In order to determine the optimal hyperparameters for the SVM, a nested grid search on each fold was implemented

Table 1 First order and second and high order texture features

\begin{tabular}{|c|c|c|c|}
\hline $\begin{array}{l}\text { Histogram- } \\
\text { derived }\end{array}$ & GLCM & GLRLM & GLSZM \\
\hline Entropy & Contrast & Short-run emphasis (SRE) & Small zone emphasis (SZE) \\
\hline Variance & Correlation & Long-run emphasis (LRE) & Large zone emphasis (LZE) \\
\hline Skewness & Energy & $\begin{array}{l}\text { Grey-level non-uniformity } \\
(G L N)\end{array}$ & $\begin{array}{l}\text { Grey-level non-uniformity } \\
(G L N)\end{array}$ \\
\hline Kurtosis & Homogeneity & $\begin{array}{l}\text { Run length non-uniformity } \\
\text { (RLN) }\end{array}$ & $\begin{array}{l}\text { Zone-size non-uniformity } \\
\text { (ZSN) }\end{array}$ \\
\hline Contrast & $\begin{array}{l}\text { Short-run emphasis } \\
\text { (SRE) }\end{array}$ & $\begin{array}{l}\text { Run percentage } \\
\text { (RP) }\end{array}$ & Zone percentage (ZP) \\
\hline Correlation & $\begin{array}{l}\text { Long-run emphasis } \\
\text { (LRE) }\end{array}$ & $\begin{array}{l}\text { Low grey-level run emphasis } \\
\text { (LGRE) }\end{array}$ & $\begin{array}{l}\text { Low grey-level zone emphasis } \\
\text { (LGZE) }\end{array}$ \\
\hline Energy & $\begin{array}{l}\text { Grey-level non-uniformity } \\
\text { (GLN) }\end{array}$ & $\begin{array}{l}\text { High grey-level run emphasis } \\
\text { (HGRE) }\end{array}$ & $\begin{array}{l}\text { High grey-level zone emphasis } \\
\text { (HGZE) }\end{array}$ \\
\hline \multirow[t]{8}{*}{ Homogeneity } & $\begin{array}{l}\text { Run length non-uniformity } \\
\text { (RLN) }\end{array}$ & $\begin{array}{l}\text { Short-run low grey-level emphasis } \\
\text { (m_SRLGE) }\end{array}$ & $\begin{array}{l}\text { Small zone low-grey level emphasis } \\
\text { (SZLGE) }\end{array}$ \\
\hline & Run percentage (RP) & $\begin{array}{l}\text { Short-run high grey-level emphasis } \\
\text { (SRHGE) }\end{array}$ & $\begin{array}{l}\text { Small zone high grey level emphasis } \\
\text { (SHZGE) }\end{array}$ \\
\hline & $\begin{array}{l}\text { Low grey-level run emphasis } \\
\text { (LGRE) }\end{array}$ & $\begin{array}{l}\text { Long-run low grey-level emphasis } \\
\text { (LRLGE) }\end{array}$ & $\begin{array}{l}\text { Large zone low grey-level emphasis } \\
\text { (LZLGE) }\end{array}$ \\
\hline & $\begin{array}{l}\text { High grey-level run emphasis } \\
\text { (HGRE) }\end{array}$ & $\begin{array}{l}\text { Long-run high-grey level emphasis } \\
\text { (LRHGE) }\end{array}$ & Large zone high grey-level (LZHGE) \\
\hline & $\begin{array}{l}\text { Short-run low grey-level emphasis } \\
\text { (SRLGE) }\end{array}$ & & Grey-level variance (GLV) \\
\hline & $\begin{array}{l}\text { Short-run high grey-level emphasis } \\
\text { (SRHGE) }\end{array}$ & & Zone-size variance (ZSV) \\
\hline & $\begin{array}{l}\text { Long-run low grey-level emphasis } \\
\text { (LRLGE) }\end{array}$ & & \\
\hline & $\begin{array}{l}\text { Long-run high grey-level emphasis } \\
\text { (LRHGE) }\end{array}$ & & \\
\hline
\end{tabular}


by specifying the parameter for gamma and $C$ in a logarithmic scale from 0.00001 to 0.001 and 1 to 1,000 , respectively. On the training partition, for each sub-dataset, the mean cross-validation accuracies of the classifier for each combination of the specified parameter value was calculated from each fold and depicted as heatmap as a function of $C$ and gamma. The parameter combination reaching the highest validation accuracy for the corresponding sub-dataset was chosen for the classification task on the test dataset.

\section{Feature selection}

To select the reduced feature set (RFS) of optimal features with superior discriminative power from the full feature set (FFS), a recursive feature elimination with cross-validation (RFECV) was performed on each of the sub-datasets. Thereby, each individual feature was ranked and the best set of features according to the classification accuracy was selected. This selection process initially included all 47 features of the dataset and then gradually removed with each iteration of those features, which contributed least to improve the classifier performance. The feature ranking was generated with regard to the number of iterations when the corresponding feature was removed and an optimal number of features was determined [24]. Subsequently, the three previously defined data subsets in the training and validation partition were reduced to the RFECV obtained optimal features, and the SVM classifier was trained and tested again on the RFS applying the same preprocessing steps and hyperparameter tuning as for the FFS.

\section{Statistical analysis}

Normally distributed data are reported as means with standard deviations otherwise as median and interquartile range (IQR). Normal distribution was assessed by using the Kolmogorov-Smirnov test. A one-way analysis of variance was performed for comparison of all texture features among malignant lesions, benign solid lesions and fat and fibroglandular tissue with post hoc Bonferroni correction (only $p$ values less than 0.0001 were considered significant). Unpaired $t$ test was used to compare all texture features between lesions (benign and malignant) versus normal tissue (fibroglandular and fat tissue). The receiver operating characteristic (ROC) curve was computed in the case of features with significant differences. The linear relationship between the different texture features in the FFS was graphically reported via a correlation matrix. For each data subset and corresponding set of features (FFS, RFS) of the validation partition, the overall and tissue-specific performance of the SVM classifier were quantified in terms of classification accuracy and metrics of the confusion matrix [25]. From the generated classification probabilities and confusion matrices, sensitivity and 1-specificity were extracted, and the area under the curve (AUC) was calculated. AUCs

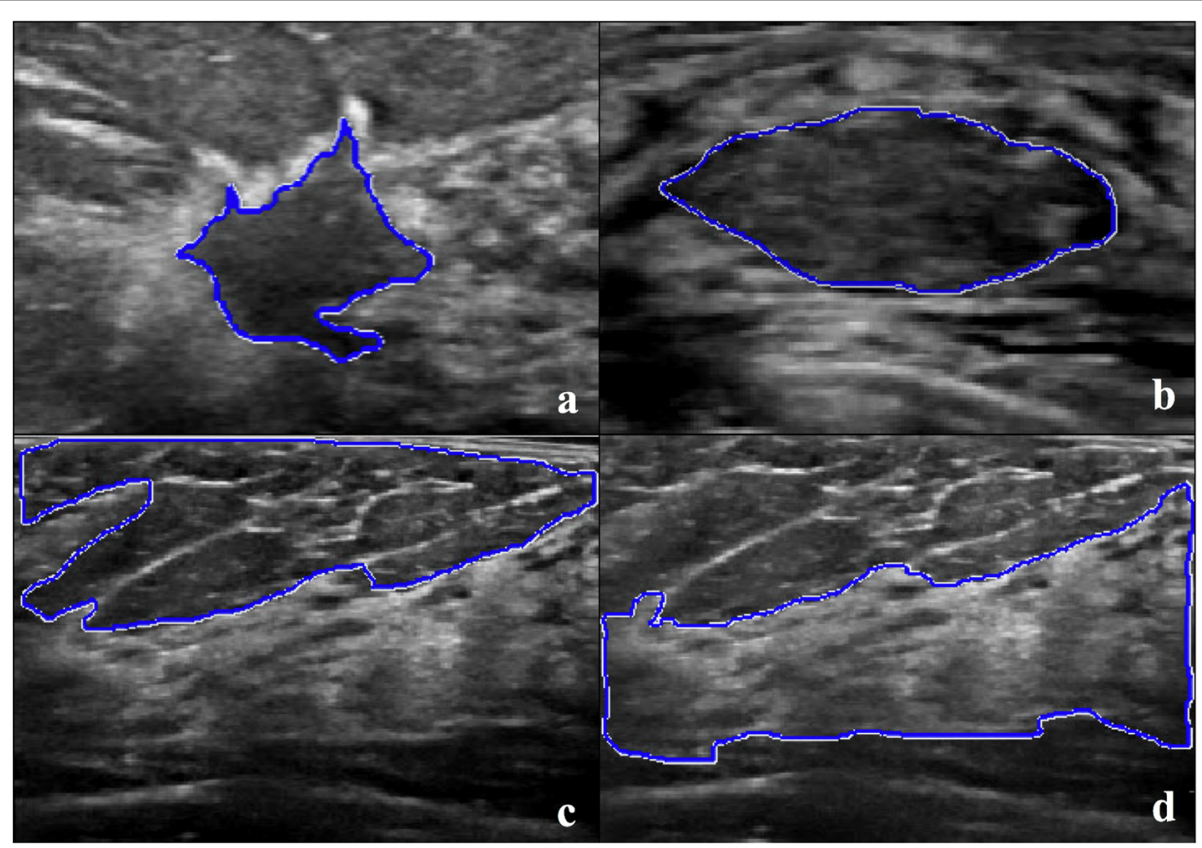

Fig. 1 Axial images obtained with automated breast ultrasound (ABUS). A region of interest was drawn freehand marking the outer edge of the lesion or the maximal continuous area of fat or fibroglandular tissue included in the image. Invasive ductal carcinoma in a 53-year-old patient undergoing screening mammography and ABUS (a). Stable benign solid lesion after a 48-month follow-up in a 35-year-old woman undergoing routine control (b). Fatty tissue (c). Fibroglandular tissue (d) 
were compared with each other according to DeLong's non-parametric test using MedCalc for Windows, version 18.2.1 (MedCalc Software, Osten, Belgium). A $p$ value of less than 0.05 was considered for significance. The inter-reader agreement for the different TA features was evaluated using the intraclass correlation coefficient (ICC) and interpreted according to the criteria by Landis and Koch [26]: an ICC of 0.41-0.60 indicated moderate agreement, an ICC of $0.61-0.80$ indicated substantial agreement and 0.81-1.0 indicated almost perfect agreement. All statistical analyses were performed with commercially available software (SPSS, release 22.0; SPSS Inc, Chicago, IL, MedCalc for Windows and d the Scikit-learn package with Python release 3.6) [27].

\section{Results}

Thirty-eight solid benign solid lesions (5 biopsy-proved fibroadenomas) and 32 malignant lesions (30 invasive ductal carcinomas, 2 invasive lobular carcinomas) were evaluated in 54 women. Nine patients had multiple benign lesions, three patients had multifocal, and one patient multicentric disease. The median maximum diameter of benign lesions was $14 \mathrm{~mm}$ (IQR $12.0-18.0 \mathrm{~mm}$, range 7-36 mm) and of malignant lesions was $14 \mathrm{~mm}$ (IQR $10.5-19.8 \mathrm{~mm}$, range $5-50 \mathrm{~mm}$ ). A total of 253 images from malignant (approximately 7 images/lesion, range 2-16), 254 images from benign lesions (approximately 6 images/lesion, range 3-16) and 108 images each for fat and fibroglandular tissue were analysed.

\section{Texture analysis}

Median ROI size was 1,312 pixels (IQR 1,161-2,461) for benign lesions, 2,220 pixels (IQR $1,638-2,839$ ) for malignant lesions, 10,529 pixels (IQR 8,074-15,205) for fatty tissue, and 14,296 pixels (IQR 12,736-19,845) for fibroglandular tissue (Fig. 1a-d). Skewness and kurtosis were the only features significantly different among the four categories $(p<0.000001)$. Texture features, which exhibited significant differences when comparing lesions versus normal tissue and malignant versus benign lesion, with corresponding AUC, are reported in Tables 2 and 3 as well as in Figs. 2 and 3, respectively. At the ROC analysis, the energy was the texture feature with the maximum AUC value in the comparison of lesions versus normal tissue $(0.86,95 \% \mathrm{CI} 0.82-0.88)$ and a total of

Table 2 Texture features that showed significantly different mean values comparing lesions (benign and malignant) versus normal tissue (fat and fibroglandular) and corresponding area under the curve (AUC)

\begin{tabular}{|c|c|c|c|c|}
\hline Feature & $\begin{array}{l}\text { Lesions (mean } \pm \text { standard } \\
\text { deviation) }\end{array}$ & $\begin{array}{l}\text { Normal tissue (mean } \pm \text { standard } \\
\text { deviation) }\end{array}$ & $p$ value & $\begin{array}{l}\text { AUC ( } 95 \% \text { confidence } \\
\text { interval) }\end{array}$ \\
\hline Entropy & $5.48 \pm 0.35$ & $5.65 \pm 0.24$ & \multirow{20}{*}{$\begin{array}{l}<0.00001 \text { for } \\
\text { all }\end{array}$} & $0.67(0.63-0.71)$ \\
\hline Variance & $153.53 \pm 23.59$ & $137.75 \pm 20.12$ & & $0.70(0.66-0.73)$ \\
\hline Contrast & $24.10 \pm 9.87$ & $40.10 \pm 12.5$ & & $0.84(0.81-0.87)$ \\
\hline Correlation & $0.88 \pm 0.06$ & $0.81 \pm 0.06$ & & $0.80(0.65-0.83)$ \\
\hline Energy & $3.4 \times 10^{-3} \pm 1.5 \times 10^{-3}$ & $2.2 \times 10^{-3} \pm 0.7 \times 10^{-3}$ & & $0.83(0.80-0.86)$ \\
\hline Homogeneity & $0.36 \pm 0.05$ & $0.33 \pm 0.04$ & & $0.72(0.69-0.76)$ \\
\hline Contrast & $26.43 \pm 12.38$ & $40.94 \pm 13.41$ & & $0.80(0.76-0.83)$ \\
\hline Correlation & $0.87 \pm 0.06$ & $0.81 \pm 0.07$ & & $0.78(0.74-0.82)$ \\
\hline Energy & $3.9 \times 10^{-3} \pm 1.4 \times 10^{-3}$ & $2.6 \times 10^{-3} \pm 0.7 \times 10^{-3}$ & & $0.86(0.82-0.88)$ \\
\hline $\begin{array}{l}\text { Homogeneity } \\
\text { (GLCM) }\end{array}$ & $0.36 \pm 0.05$ & $0.33 \pm 0.04$ & & $0.71(0.66-0.74)$ \\
\hline GLN (GLCM) & $78.42 \pm 77.69$ & $156.28 \pm 104.76$ & & $0.79(0.76-0.83)$ \\
\hline RLN (GLCM) & $1,870.43 \pm 1,792.01$ & $4,085.00 \pm 2,628.83$ & & $0.82(0.79-0.85)$ \\
\hline LRHGE (GLCM) & $1,706.53 \pm 209.23$ & $1,635.50 \pm 197.26$ & & $0.61(0.57-0.65)$ \\
\hline SRE (GLRLM) & $0.91 \pm 0.02$ & $0.92 \pm 0.04$ & & $0.69(0.65-0.73)$ \\
\hline LRE (GLRLM) & $1.47 \pm 0.17$ & $1.39 \pm 0.12$ & & $0.65(0.61-0.69)$ \\
\hline GLN (GLRLM) & $78.53 \pm 77.67$ & $156.36 \pm 104.73$ & & $0.79(0.76-0.83)$ \\
\hline RLN (GLRLM) & $1,871.42 \pm 1,786.51$ & $4,080.90 \pm 2,622.01$ & & $0.82(0.79-0.85)$ \\
\hline LRHGE (GLRLM) & $1,677.30 \pm 196.74$ & $1,598.70 \pm 168.52$ & & $0.61(0.57-0.65)$ \\
\hline LZE & $5.38 \pm 3.34$ & $4.07 \pm 2.38$ & & $0.66(0.62-0.70)$ \\
\hline HGZE & $1,221.08 \pm 45.52$ & $1,191.37 \pm 86.03$ & & $0.67(0.63-0.71)$ \\
\hline
\end{tabular}

GLCM Grey-level co-occurrence matrix, GLN Grey-level non-uniformity, RLN Run length non-uniformity, LRHGE Long-run high grey-level emphasis, GLRLM Grey-level run length matrix, SRE Short-run emphasis, LRE Long-run emphasis, LZE Large zone emphasis, HGZE High grey-level zone emphasis 
Table 3 Texture features that showed significantly different mean values comparing malignant versus benign solid lesions and corresponding area under the curve (AUC)

\begin{tabular}{|c|c|c|c|c|}
\hline Feature & $\begin{array}{l}\text { Malignant lesions (mean } \pm \text { standard } \\
\text { deviation) }\end{array}$ & $\begin{array}{l}\text { Benign lesions (mean } \pm \text { standard } \\
\text { deviation) }\end{array}$ & $p$ value & $\begin{array}{l}\text { AUC ( } 95 \% \text { confidence } \\
\text { interval) }\end{array}$ \\
\hline Entropy & $5.28 \pm 0.38$ & $5.67 \pm 0.16$ & \multirow[t]{12}{*}{$<0.00001$ for all } & $0.86(0.82-0.89)$ \\
\hline Skewness & $0.74 \pm 0.33$ & $0.54 \pm 0.41$ & & $0.66(0.61-0.70)$ \\
\hline Kurtosis & $0.53 \pm 0.67$ & $0.31 \pm 0.66$ & & $0.61(0.56-0.65)$ \\
\hline Contrast & $24.45 \pm 10.61$ & $28.40 \pm 13.66$ & & $0.58(0.53-0.63)$ \\
\hline GLN (GLCM) & $96.33 \pm 87.47$ & $60.58 \pm 61.73$ & & $0.67(0.62-0.71)$ \\
\hline RLN (GLCM) & $2,218.97 \pm 1,834.77$ & $1,523.25 \pm 1,681.38$ & & $0.66(0.62-0.71)$ \\
\hline HGRE (GLCM) & $1,163.54 \pm 20.49$ & $1,171.63 \pm 19.35$ & & $0.62(0.57-0.67)$ \\
\hline SRHGE (GLCM) & $1,067.79 \pm 32.58$ & $1,080.79 \pm 29.69$ & & $0.63(0.58-0.67)$ \\
\hline GLN (GLRLM) & $96.43 \pm 87.45$ & $60.70 \pm 61.72$ & & $0.67(0.62-0.71)$ \\
\hline RLN (GLRLM) & $2,218.89 \pm 1,828.26$ & $1,525.31 \pm 1,677.17$ & & $0.67(0.62-0.71)$ \\
\hline HGRE (GLRLM) & $1,165.00 \pm 20.59$ & $1,173.06 \pm 19.57$ & & $0.62(0.57-0.67)$ \\
\hline $\begin{array}{l}\text { SRHGE } \\
\text { (GLRLM) }\end{array}$ & $1,069.20 \pm 33.13$ & $1,082.28 \pm 29.75$ & & $0.62(0.58-0.67)$ \\
\hline
\end{tabular}

GLN Grey-level non-uniformity, GLCM Grey-level co-occurrence matrix, RLN Run length non-uniformity, HGRE High grey-level run emphasis, SRHGE Short-run high grey-level emphasis, GLN Grey-level non-uniformity, GLRLM Grey-level run length matrix

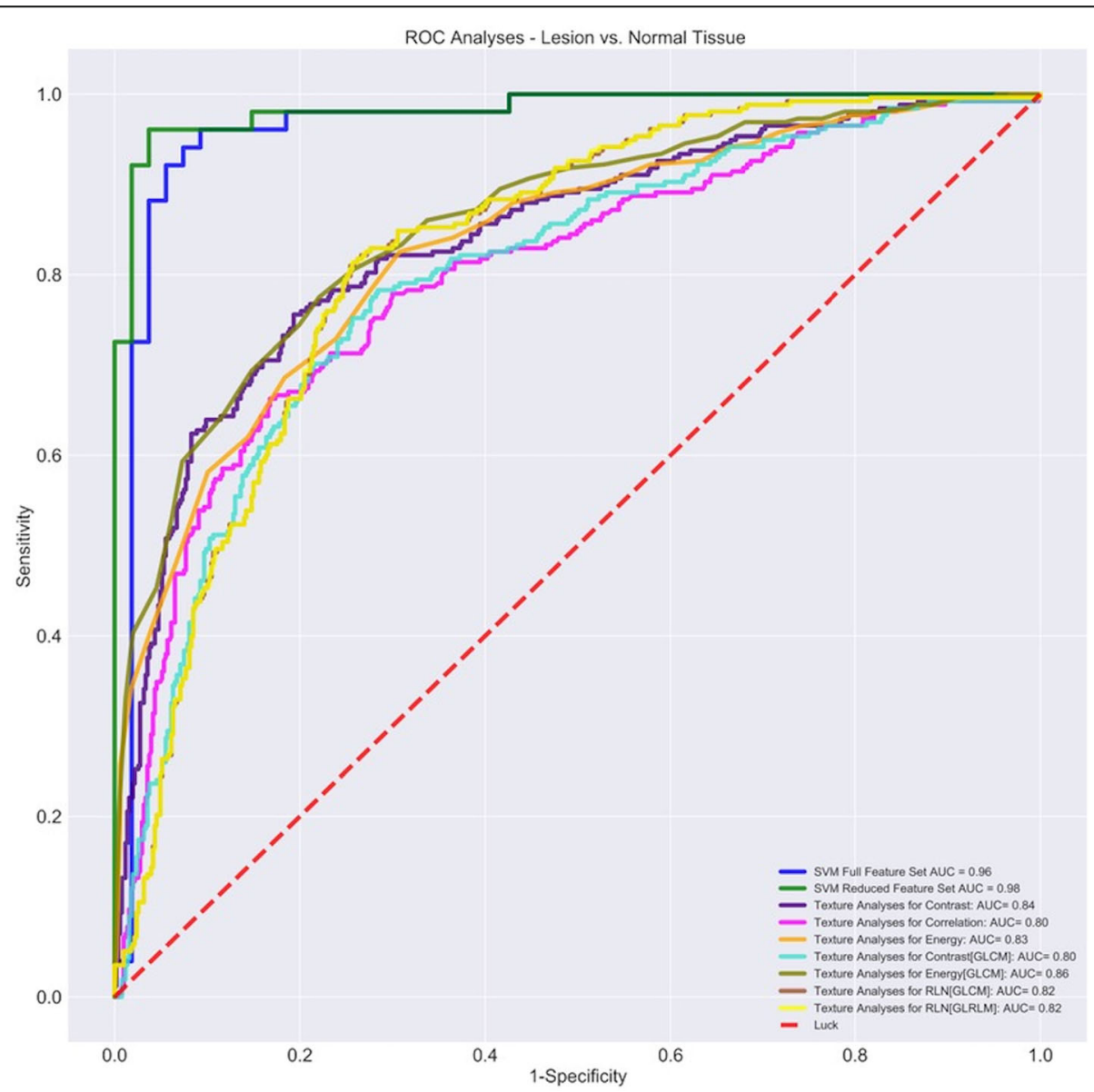

Fig. 2 Receiver operating characteristic (ROC) curves of texture analysis features with the highest area under the curve values (Table 2) as well as ROC curves obtained with machine learning support vector machine (SVM) algorithm with full and reduced features when used to compare lesions versus normal tissue on automated breast ultrasound 


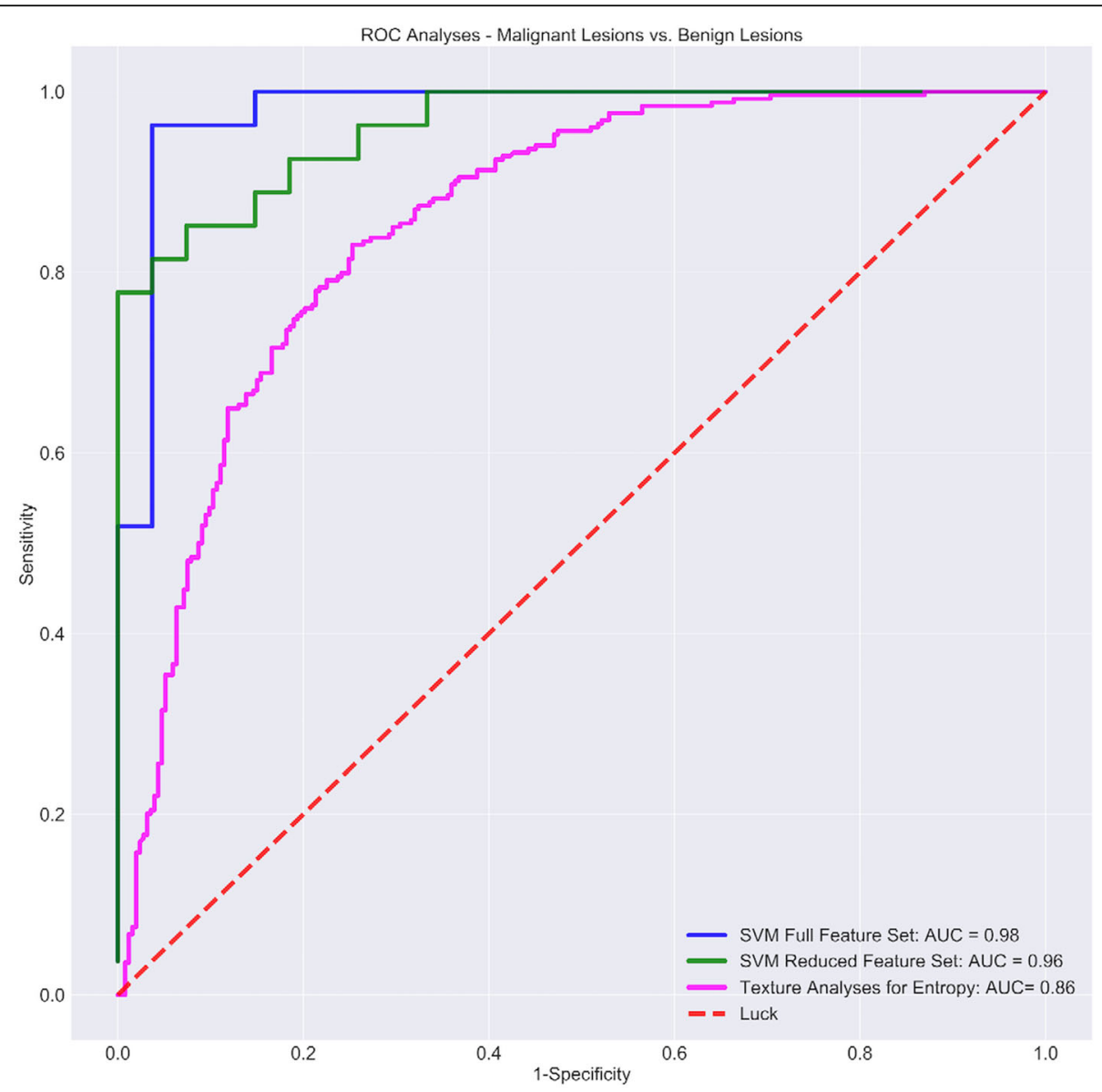

Fig. 3 Receiver operating characteristic (ROC) curve obtained from the texture analysis for entropy as well as ROC curves obtained with machine learning support vector machine (SVM) algorithm with full and reduced features when used to compare malignant versus benign solid breast lesions on automated breast ultrasound

seven features had AUC values equal or superior to 0.80 . Entropy was the texture feature with the maximum AUC value $(0.86,95 \%$ CI $0.82-0.89)$ in the comparison between benign versus malignant lesions and the only one with an AUC value superior to 0.80. The ICC showed substantial to an almost perfect agreement in the measure of all texture features $(\mathrm{ICC}=0.65-0.96$, Additional file 1: Table S1).

\section{Machine learning}

Correlation matrices for each sub-dataset (lesion versus tissue and benign versus malignant) with the FFS were displayed in Additional file 1: Figure S1A and S1B, respectively, showing significant co-correlation of several features among the higher-order features in A.

\section{Sub-dataset (i): solid lesions versus normal tissue}

The validation dataset included 105 images (54 images of lesions and 51 images of normal tissue). For the classification of lesions versus normal tissue, the optimal hyperparameters for the FFS accounted 1,000 and 0.001 for $C$ and gamma, respectively (Additional file 1: Figure S2A). Classification accuracies of $92.8 \%$ on the training set and of $93.3 \%$ on the validation set (Table 4) were reached, with $3.8 \%$ of all images in the validation partition being falsely classified as normal tissue and $2.9 \%$ as lesion instead of normal tissue (Table 5). ROC analyses revealed an AUC of 0.96 (95\% CI 0.89-0.98) for the validation set (Fig. 2). After training and validating, the SVM classifier on the FFS, a recursive feature elimination with cross-validation, was performed determining 14 features (Fig. 4a) as optimal features, composing the RFS. For the RFS, a correlation matrix was generated and the optimal hyperparameters were determined as $C=1,000$ and gamma $=0.00001$ (Additional file 1: Figures S1C and S2B). Training and validation accuracies were $91.3 \%$ and $94.4 \%$, respectively, with $1.9 \%$ of all images being falsely classified as lesions and 3.8\% as normal tissue (Tables 4 and 5). The AUC for the RFS measured 0.98 (Fig. 2). For all showed texture feature-derived ROC curves (only features with AUC values equal or superior to 0.80 ) compared to the via ML- 
Table 4 Area under the curve (AUC), accuracy, sensitivity, and specificity achieved with the validation set in the classification of lesions versus normal tissue and malignant versus benign solid lesions using the full texture feature set and the reduced feature set

\begin{tabular}{|c|c|c|c|c|}
\hline Sub-dataset & AUC (95\% confidence interval) & Accuracy (\%) & Sensitivity (\%) & Specificity (\%) \\
\hline \multicolumn{5}{|c|}{ Lesions versus normal tissue } \\
\hline Full feature set & $0.96(0.89-0.98)$ & 93.3 & 92.6 & 94.1 \\
\hline Reduced feature set & $0.98(0.92-0.99)$ & 94.4 & 96.3 & 92.1 \\
\hline \multicolumn{5}{|c|}{ Malignant versus benign lesions } \\
\hline Full feature set & $0.98(0.81-0.99)$ & 90.7 & 85.2 & 96.3 \\
\hline Reduced feature set & $0.96(0.90-0.99)$ & 87.1 & 81.5 & 92.6 \\
\hline
\end{tabular}

derived ROC curve, $p$ values were $<0.05$ (ranging from 0.003 to 0.02 ), indicating a significant difference between the areas. The two lesions incorrectly classified as normal tissue were one malignant and one benign (Fig. 5).

\section{Sub-dataset (ii): malignant versus benign solid lesions}

The validation dataset included 54 images (27 images of lesions and 27 images of normal tissue). For the classification of the malignant versus benign solid lesions, the optimal hyperparameters for the full feature set accounted 100 and 0.001 for $C$ and gamma (Additional file 1: Figure S2C). The accuracy on the training set measured $89.0 \%$ and on the validation set $90.7 \%$ with $7.4 \%$ of all lesions being falsely classified as benign lesions and $1.9 \%$ falsely as malignant (Tables 4 and 5 , Fig. 5). The AUC measured 0.98 (Fig. 3). After RFECV, a correlation matrix for the reduced feature set of 25 features (Fig. 4b) was generated applying the optimal hyperparameters of $C=1,000$ and gamma $=0.001$ (Additional file 1: Figures S1D and S2D). The classification accuracy for the RFS was $89.0 \%$ on the training and $87.1 \%$ on the validation partition (Table 4). After feature reduction, the false-positive rate of malignant lesions being falsely classified as benign increased to 9.2 $\%$ and AUC decreased to 0.96 (Fig. 3). The ROC curve for entropy, derived from texture analysis, was significantly different $(p=0.003)$ from the via ML-derived ROC curve.

\section{Discussion}

In the current study, we demonstrated that texture feature analysis of breast imaging findings in ABUS examinations might be used to differentiate malignant and benign solid lesions as well as normal tissue of the breast with high accuracy. We also showed that ML applied to texture data might be superior compared to the statistical analysis of single texture features.

Although the interrelation between the data derived from TA and potential underlying biological properties has not yet been resolved, a number of previous works have investigated the use of TA to quantify spatial heterogeneity of benign and malignant lesions in images acquired with different modalities [9-16]. A limited number of studies explored the use of TA or

Table 5 Results in the validation set for the classification of lesions versus normal tissue and malignant versus benign solid lesions using the full texture feature and the reduced feature set

\begin{tabular}{|c|c|c|c|}
\hline & Predicted & & \\
\hline \multirow[t]{14}{*}{ Actual } & Lesions versus normal tissue $(n=105)$ & Lesions (\%) & Normal tissue (\%) \\
\hline & Lesions $(n=54)$ & & \\
\hline & Full feature set & $50(92.6)$ & $4(7.4)$ \\
\hline & Reduced feature set & $52(96.3)$ & $2(3.7)$ \\
\hline & Normal tissue $(n=51)$ & & \\
\hline & Full feature set & $3(5.9)$ & $48(94.1)$ \\
\hline & Reduced feature set & $4(7.8)$ & $47(92.2)$ \\
\hline & Malignant versus benign lesions $(n=54)$ & Malignant (\%) & Benign (\%) \\
\hline & Malignant (tot $=27$ ) & & \\
\hline & Full feature set & $23(85.2)$ & $4(14.8)$ \\
\hline & Reduced feature set & $22(81.5)$ & $5(18.5)$ \\
\hline & Benign (tot $=27$ ) & & \\
\hline & Full feature set & $1(3.7)$ & $26(96.3)$ \\
\hline & Reduced feature set & $2(7.4)$ & $25(92.6)$ \\
\hline
\end{tabular}




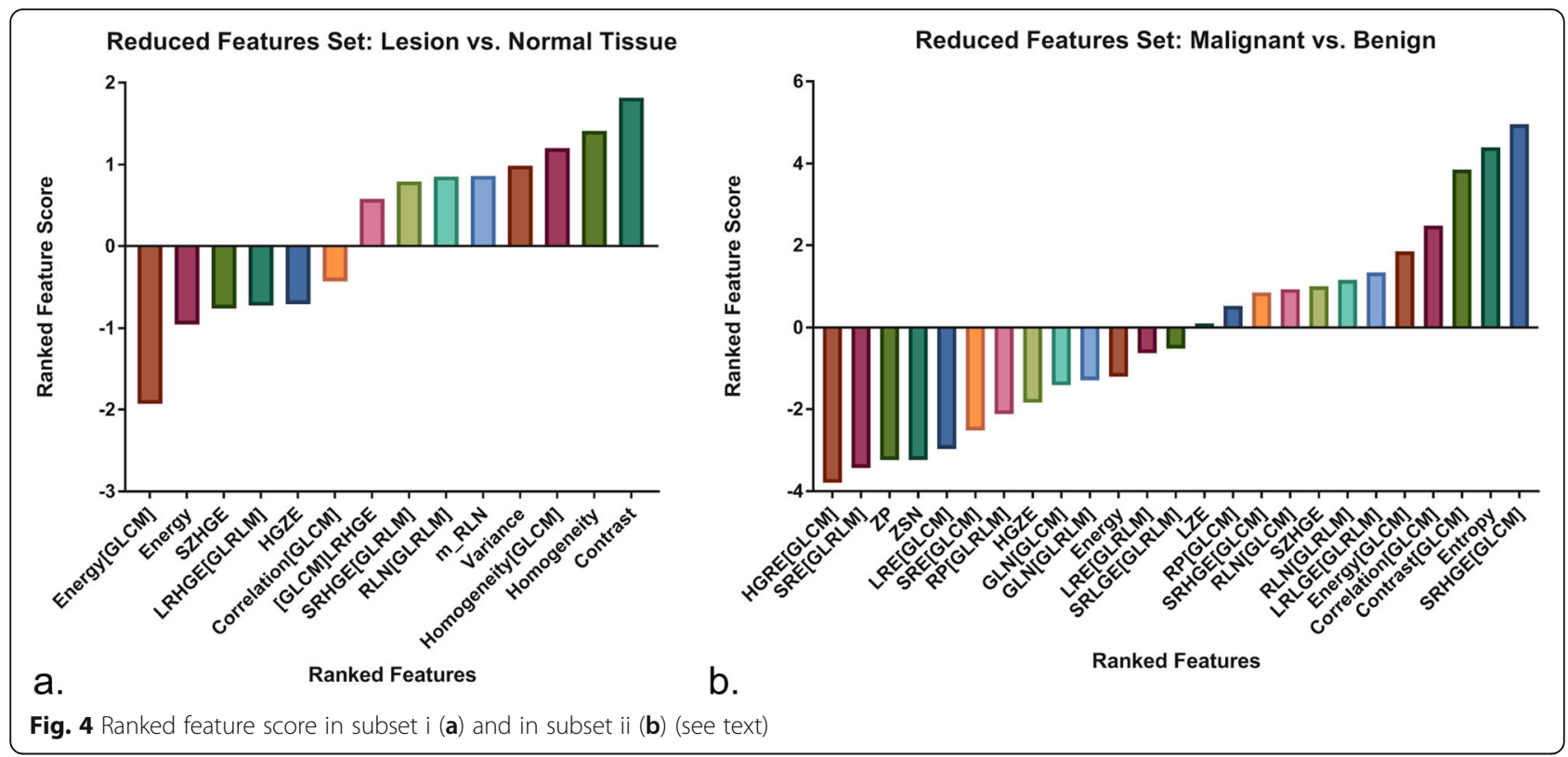

ML in ultrasound imaging for characterisation of breast lesions [28-30]. Indeed, the application of TA in conventional B-mode imaging is hindered by variations of scanning parameters that can determine unwanted variations in the assessment of texture features. Standardised acquisitions in ABUS can in part overcome these limitations.

In our study, a number of texture features exhibited significant differences when used to distinguish solid breast lesions from normal tissue as well as malignant from benign solid lesions with a relatively high AUC up to 0.86 in both cases. ML offers the possibility to train algorithms to recognise patterns of data derived from the analysis of multiple texture features instead of referring to a single feature. The use of a ML model based on the SVM algorithm with radial basis function determined an increase in the AUC to a maximum of 0.96 in the differentiation of lesions versus normal tissue as well as in the differentiation of malignant versus benign lesions with a maximal accuracy of $94.4 \%$ and $90.7 \%$, respectively. The use of recursive feature selection in the test datasets for differentiation of lesions versus normal tissue resulted in an increase in the AUC to 0.98 whereas for malignant versus benign lesions, the AUC slightly decreased to 0.96 . Moreover, application of the reduced feature sets resulted in nearly the same training accuracies for the training data and even a slightly higher accuracy of $94.4 \%$ for the test dataset differentiating lesions versus normal tissue. These excellent performances for the full as well as for the reduced feature sets and the associated low amount of overfitting emphasise the robustness and

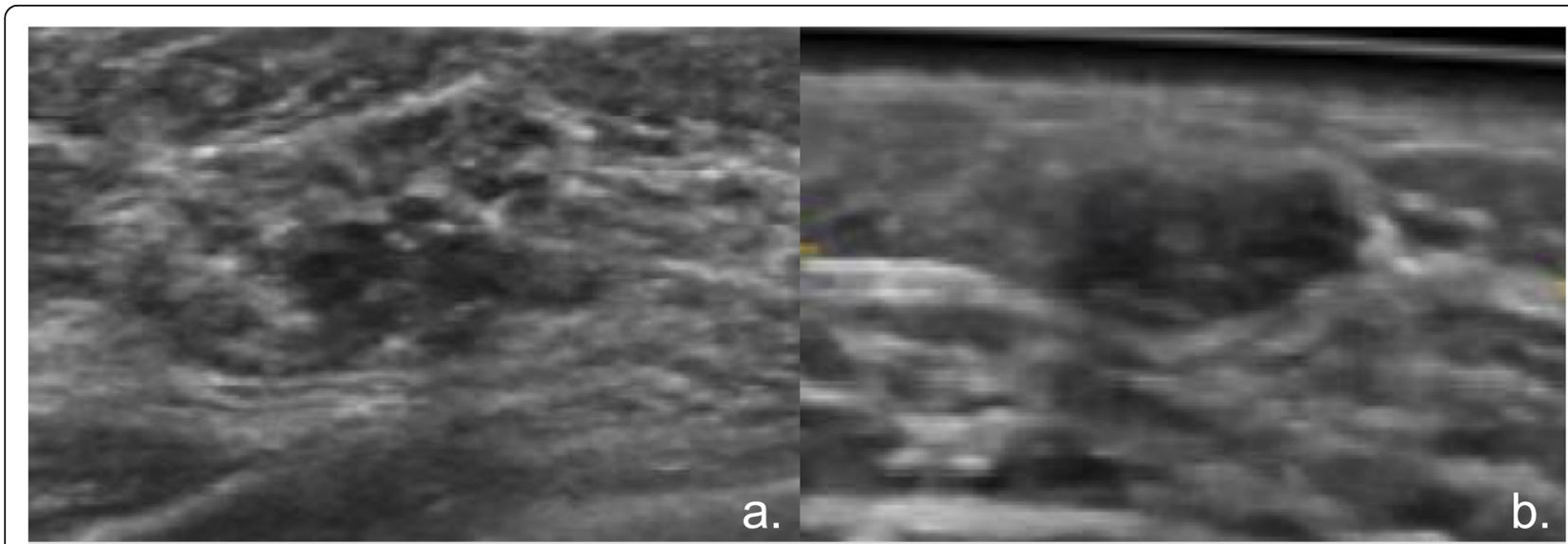

Fig. 5 Lesions falsely classified as normal tissue using machine learning with the reduced feature set but correctly classified with the full feature set: invasive ductal carcinoma (maximal diameter $10 \mathrm{~mm}$ ) in a 74-year-old patient (a) and fibroadenoma (maximal diameter $9 \mathrm{~mm}$ ) in a $46-y e a r-$ old patient (b). Lesion (a) was also falsely classified as benign in the comparison between malignant and benign lesions 
stability of the applied ML model. In many cases, overfitting occurs when the ML algorithm is trained in a too-large extent with details and noise negatively affecting the performance on real-world data. In order to minimise overfitting, the SVM on our study was trained via cross-validation, dividing the training data into subsets of equal size, which also provided advantages with respect to the limited number of data points. In addition, the robustness can be accounted, to some extent, that special interest was put into the acquisition of balanced datasets, and no oversampling techniques were applied to synthetically generate data [31].

Previous studies reported that the use of supplemental ABUS in breast cancer screening programmes causes an increase of the recall rate $[6,32]$. Moreover, misinterpretation of lesions along with the presence of multiple distracting lesions are determining factors in the case of undiagnosed cancers at supplemental screening ultrasonography [33]. Although computer-aided-detection software for ABUS offers the potential to improve radiologists' performances in detecting breast cancer, characterisation of the imaging findings remains a major issue [34, 35]. In a recent study, van Zelst et al. [35] showed that the AUCs between conventional $A B U S$ reading and computer-aided-detectionbased reading performed by eight radiologists with variable years of ABUS experience was not significantly different (0.82 and 0.83, respectively). The combined use of CAD software with algorithms, that enable TA combined to ML, might overcome the relative limitations of the two approaches (i.e., the limited specificity of CAD and the necessity for aided-detection in TA combined to ML). Although the differentiation of breast lesions from normal breast tissue was quite straightforward in our cases, we decided to include also this evaluation considering the potential role of ML algorithms integrated in the software for ABUS image evaluation. A maximal accuracy of 94.4 was observed when comparing normal tissue versus breast lesions. More important, in our study, a very high specificity (maximal 96.3\%) was achieved in the comparison of benign versus malignant lesions using ML.

Our study has some major limitations. First, the underpowered analysis due to the limited number of cases is included. Nevertheless, the purpose of our pilot study was to present a possible approach for the evaluation of breast imaging findings in ABUS and to enhance some differences when TA information alone or in conjunction with $M L$ is used. A possibly prospective study including a higher number of cases is necessary to confirm our results. Second, the high number of evaluated images was derived from a relatively low number of different lesions that could have biased the results. Nevertheless, both malignant and benign solid lesions were collected from the general female population referred to our department for screening or follow-up examination of known lesions presumably forming a sufficient representative group of breast solid lesions. Third, we did not compare the performance of TA and ML with the performance of radiologists with different levels of experience, which was beyond the scope of this study. Also, although the inter-reader agreement for the assessment of the texture feature measurements was evaluated only in part of the lesions, we could demonstrate a high reproducibility of the measurements for all features.

In conclusion, our pilot study demonstrated that TA in combination with ML might represent a useful diagnostic tool in the evaluation of ABUS findings. Applying ML techniques to texture features might be superior compared to analysis of single texture features. A prospective study including a higher number of cases is necessary to confirm our results.

\section{Abbreviations}

ABUS: Automated breast ultrasound; AUC: Area under the curve; FFS: Full feature set; GLCM: Grey-level co-occurrence matrix; GLRLM: Grey-level run length matrix; GLSZM: Grey-level size zone matrix; HHUS: Hand-held ultrasound; ICC: Intraclass correlation coefficient; IQR: Interquartile range; ML: Machine learning; RFECV: Recursive feature elimination with crossvalidation; RFS: Reduced feature set; ROC: Receiver operating characteristic; ROI: Region of interest; SVM: Support vector machine; TA: Texture analysis

\section{Authors' contributions}

$\mathrm{MM}, \mathrm{AC}$, and $\mathrm{AB}$ contributed substantially to the acquisition, analysis, and interpretation of the data, wrote the main manuscript text, and prepared the figures and tables. CR, ASB, NB, MCW, MWW, and TF contributed to the acquisition, analysis, and interpretation of the data. All authors edited the manuscript. All authors reviewed and approved the final manuscript.

\section{Funding}

MM was financially supported by a grant from the Promedica Foundation. NB was financially supported by the "Filling the Gap" grant from the University of Zurich.

\section{Availability of data and materials}

The datasets used and/or analysed during the current study are available from the corresponding author on reasonable request.

\section{Ethics approval and consent to participate}

This study protocol was approved by the Cantonal Ethics Committee of Zürich, Switzerland (Approval Number: 2016-00064) and the need for informed consent was waived.

\section{Consent for publication}

Not applicable.

\section{Competing interests}

The authors of this manuscript declare relationships with the following companies: the corresponding author (MM) has received honoraria from GE Healthcare for giving lectures and for moderating workshops.

Received: 18 April 2019 Accepted: 28 August 2019

Published online: 01 November 2019

\section{Additional file}

Additional file 1: Table S1. Inter-reader agreement for the different TA features was evaluated using the intraclass correlation coefficient (ICC). Figure S1. Correlation matrix generated from the full texture feature set for the sub-datasets lesions versus normal tissue (A) and malignant versus benign solid lesions (B) as well as from the corresponding reduced feature set (C) and (D). A significant co-correlation of several features is 
present in particular among the higher order features in A (e.g. SRE[GLCM] and HGRE[GLCM]) as possible reflection of underlying common biological properties. Figure $\mathbf{S 2}$. Heatmaps depicting the optimal hyperparameters for the full feature (A, B) and the reduced feature training datasets $(C, D)$. The hyperparameter tuning was implemented via nested grid search on the SVM classifier by specifying the parameter for gamma and (C) in a logarithmic scale from 0.00001 to 0.001 and 1 to 1000, respectively. (DOCX $4241 \mathrm{~kb}$ )

Received: 18 April 2019 Accepted: 28 August 2019

\section{Published online: 01 November 2019}

\section{References}

1. Bae MS, Moon WK, Chang JM et al (2014) Breast cancer detected with screening US: reasons for nondetection at mammography. Radiology 270: 369-377 https://doi.org/10.1148/radiol.13130724

2. Hooley RJ, Greenberg KL, Stackhouse RM, Geisel JL, Butler RS, Philpotts LE (2012) Screening US in patients with mammographically dense breasts: initial experience with Connecticut Public Act 09-41. Radiology 265:59-69 https://doi.org/10.1148/radiol.12120621

3. Kolb TM, Lichy J, Newhouse JH (2002) Comparison of the performance of screening mammography, physical examination, and breast US and evaluation of factors that influence them: an analysis of 27,825 patient evaluations. Radiology 225:165-175 https:/doi.org/10.1148/radiol.2251011667

4. Berg WA, Zhang Z, Lehrer D et al (2012) Detection of breast cancer with addition of annual screening ultrasound or a single screening MRI to mammography in women with elevated breast cancer risk. JAMA 307:1394-1404 https://doi.org/10.1001/jama.2012.388

5. Weigert J, Steenbergen S (2012) The Connecticut Experiment: the role of ultrasound in the screening of women with dense breasts. Breast J 18:517-522. https://doi.org/10.1111/tbj.12003

6. Brem RF, Tabár L, Duffy SW et al (2015) Assessing improvement in detection of breast cancer with three-dimensional automated breast US in women with dense breast tissue: the Somolnsight Study. Radiology 274:663-673 https://doi.org/10.1148/radiol.14132832

7. Ganeshan B, Miles KA (2013) Quantifying tumour heterogeneity with CT. Cancer Imaging 13:140-149 https://doi.org/10.1102/1470-7330.2013.0015

8. Lubner MG, Smith AD, Sandrasegaran K, Sahani DV, Pickhardt PJ (2017) CT texture analysis: definitions, applications, biologic correlates, and challenges. Radiographics 37:1483-1503 https://doi.org/10.1148/rg.2017170056

9. Becker AS, Ghafoor S, Marcon M et al (2017) MRI texture features may predict differentiation and nodal stage of cervical cancer: a pilot study. Acta Radiol Open 6:2058460117729574 https://doi.org/10.1177/ 2058460117729574

10. Ganeshan B, Panayiotou E, Burnand K, Dizdarevic S, Miles K (2012) Tumour heterogeneity in non-small cell lung carcinoma assessed by $C T$ texture analysis: a potential marker of survival. Eur Radiol 22:796-802 https://doi. org/10.1007/s00330-011-2319-8

11. Ng F, Ganeshan B, Kozarski R, Miles KA, Goh V (2013) Assessment of primary colorectal cancer heterogeneity by using whole-tumor texture analysis: contrast-enhanced CT texture as a biomarker of 5-year survival. Radiology 266:177-184 https://doi.org/10.1148/radiol.12120254

12. Park HJ, Lee SM, Song JW et al (2016) Texture-based automated quantitative assessment of regional patterns on initial $C T$ in patients with idiopathic pulmonary fibrosis: relationship to decline in forced vital capacity. AJR Am J Roentgenol 207:976-983 https://doi.org/10.2214/AJR.16.16054

13. Simpson AL, Adams LB, Allen PJ et al (2015) Texture analysis of preoperative $C T$ images for prediction of postoperative hepatic insufficiency: a preliminary study. J Am Coll Surg 220:339-346 https://doi.org/10.1016/j. jamcollsurg.2014.11.027

14. Yip C, Landau D, Kozarski R et al (2014) Primary esophageal cancer: heterogeneity as potential prognostic biomarker in patients treated with definitive chemotherapy and radiation therapy. Radiology 270:141-148 https://doi.org/10.1148/radiol.13122869

15. Zhang H, Graham CM, Elci O et al (2013) Locally advanced squamous cell carcinoma of the head and neck: CT texture and histogram analysis allow independent prediction of overall survival in patients treated with induction chemotherapy. Radiology 269:801-809 https:// doi.org/10.1148/radiol.13130110
16. Park YS, Seo JB, Kim N et al (2008) Texture-based quantification of pulmonary emphysema on high-resolution computed tomography: comparison with density-based quantification and correlation with pulmonary function test. Invest Radiol 43:395-402 https://doi.org/10.1097/ RLI.0b013e31816901c7

17. Erickson BJ, Korfiatis P, Akkus Z, Kline TL (2017) Machine learning for medical imaging. Radiographics 37:505-515 https://doi.org/10.1148/rg. 2017160130

18. D'Orsi CJ, Sickles EA, Mendelson EB, Morris EA et al (2013) ACR BI-RADS Atlas, Breast Imaging Reporting and Data System. American College of Radiology, Reston

19. Vallières M, Freeman CR, Skamene SR, El Naqa I (2015) A radiomics model from joint FDG-PET and MRI texture features for the prediction of lung metastases in soft-tissue sarcomas of the extremities. Phys Med Biol 60: 5471-5496 https://doi.org/10.1088/0031-9155/60/14/5471

20. Becker AS, Wagner MW, Wurnig MC, Boss A (2017) Diffusion-weighted imaging of the abdomen: impact of b-values on texture analysis features. NMR Biomed 30 https://doi.org/10.1002/nbm.3669

21. Mayerhoefer ME, Szomolanyi P, Jirak D et al (2009) Effects of magnetic resonance image interpolation on the results of texture-based pattern classification: a phantom study. Invest Radiol 44:405-411 https://doi.org/10. 1097/RLI.0b013e3181a50a66

22. Mayerhoefer ME, Szomolanyi P, Jirak D, Materka A, Trattnig S (2009) Effects of MRI acquisition parameter variations and protocol heterogeneity on the results of texture analysis and pattern discrimination: an application-oriented study. Med Phys 36:1236-1243 https://doi.org/10.1118/1.3081408

23. Hinzpeter R, Wagner MW, Wurnig MC, Seifert B, Manka R, Alkadhi H (2017) Texture analysis of acute myocardial infarction with $C T$ : first experience study. PLoS One https://doi.org/10.1371/journal.pone.0186876

24. Guyon I, Weston J, Barnhill S, Vapnik V (2002) Gene selection for cancer classification using support vector machines. Mach Learn 46:389-422 https://doi.org/10.1023/A:1012487302797

25. Beleites C, Neugebauer U, Bocklitz T, Krafft C, Popp J (2013) Sample size planning for classification models. Anal Chim Acta 760:25-33 https://doi. org/10.1016/j.aca.2012.11.007

26. Landis JR, Koch GG (1977) The measurement of observer agreement for categorical data. Biometrics 33:159-174

27. Pedregosa F, Varoquaux G, Gramfort A et al (2011) Scikit-learn: machine learning in Python. J Mach Learn Res 12:2825-2830

28. Alvarenga AV, Pereira WC, Infantosi AF, Azevedo CM (2007) Complexity curve and grey level co-occurrence matrix in the texture evaluation of breast tumor on ultrasound images. Med Phys 34:379-387 https://doi.org/ 10.1118/1.2401039

29. Liao YY, Tsui PH, Li CH et al (2011) Classification of scattering media within benign and malignant breast tumors based on ultrasound texture-featurebased and Nakagami-parameter images. Med Phys 38:2198-2207 https:// doi.org/10.1118/1.3566064

30. Becker AS, Mueller M, Stoffel E, Marcon M, Ghafoor S, Boss A (2018) Classification of breast cancer in ultrasound imaging using a generic deep learning analysis software: a pilot study. Br J Radiol 91:20170576 https://doi. org/10.1259/bjr.20170576

31. Chawla NV, Bowyer KW, Hall LO, Kegelmeyer WP (2002) SMOTE: synthetic minority over-sampling technique. J Artif Intell Res 16:321-357

32. Giuliano V, Giuliano C (2013) Improved breast cancer detection in asymptomatic women using 3D-automated breast ultrasound in mammographically dense breasts. Clin Imaging 37:480-486 https://doi.org/ 10.1016/j.clinimag.2012.09.018

33. Song SE, Cho N, Chu A et al (2015) Undiagnosed breast cancer: features at supplemental screening US. Radiology 277:372-380 https://doi.org/10.1148/ radiol.2015142960

34. van Zelst JCM, Tan T, Platel B et al (2017) Improved cancer detection in automated breast ultrasound by radiologists using computer aided detection. Eur J Radiol 89:54-59 https://doi.org/10.1016/j.jrad.2017.01.021

35. van Zelst JCM, Tan T, Clauser P et al (2018) Dedicated computer-aided detection software for automated 3D breast ultrasound; an efficient tool for the radiologist in supplemental screening of women with dense breasts. Eur Radiol 28:2996-3006. https://doi.org/10.1007/s00330-017-5280-3

\section{Publisher's Note}

Springer Nature remains neutral with regard to jurisdictional claims in published maps and institutional affiliations. 